\title{
Determinants of exclusive breastfeeding practices in Ethiopia
}

Tewodros Alemayehu, Jemal Haidar, Dereje Habte

\begin{abstract}
Background: Despite the demonstrated benefits of breast milk, the prevalence of breastfeeding, in-particular exclusive breastfeeding (EBF), in many developing countries including Ethiopia is lower than the international recommendation of EBF for the first six months of life

Objective: To assess the practice of EBF and explore its determinants in Ethiopia and provide policy makers and NGOs with relevant information for future planning and interventions.

Methods: Raw data collected from nine regions and two city administrations using stratified cluster sampling method by the Ethiopian Demographic Health Survey (EDHS) 2005 were used to study the practice and determinants of EBF countrywide. Analysis was based on children whose age was less than six months and alive at the time of interview that was extracted from the women's database.

Results: The overall rates of exclusive and full breastfeeding were $49.0 \%$ and $68.2 \%$ respectively. Maternal education, marital status, wealth index and age of the child were closely associated with EBF practices, nonetheless, in the hierarchical analysis; being not married, middle/ richer/ richest wealth index, and child age 0-1and 2-3 month were retained as the predictors of $\mathrm{EBF}(\mathrm{P}<0.05)$.

Conclusion: A range of maternal and child health attributes such as marital status, economical status and child age were found to influence the practice of EBF in Ethiopia. Actions to empower women and promotion of EBF campaign are recommended to achieve the fourth millennium development goal. [Ethiop.J.Health Dev. 2009;23(1):12-18]
\end{abstract}

\section{Introduction}

World Health Organization (WHO) and United Nations Children's Fund (UNICEF) recommend that all mothers should breastfeed their children exclusively for the first 6 months and thereafter they should continue to breastfeed for as long as the mother and child wish, and both appropriate and sufficient weaning food should be added after six months of life $(1,2)$. WHO estimates that worldwide only $35 \%$ of children between birth and their fifth month are breastfed exclusively (1). In order to achieve the Millennium Development Goal of reduction of child mortality, infant breastfeeding has been identified as one of the major intervention areas both globally and nationally $(3,4)$. Extensive research in various countries has provided evidence that breastfeeding has clear health benefits for infants as well as the mother. Infants who have been breastfed optimally have reduced risk of common childhood illnesses such as gastrointestinal and respiratory infections, otitis media, atopic eczema, and allergy during childhood (2, 5, 6).

In resource poor countries, where the negative impact of HIV/AIDS is high, exclusive breastfeeding for the first six months has greater benefit than mixed feeding or formula feeding for the prevention of mother to child transmission of HIV (7).

Exclusive breastfeeding (EBF) has been defined by WHO as the situation where 'the infant has received only breast milk from his/her mother or a wet nurse, or expressed breast milk and no other liquids, or solids, with the exception of drops or syrups consisting of vitamins, minerals supplements, or medicines (8). EBF is adequate in quality as well as quantity in terms of energy, protein, nutrients, water etc. for an infant's need under six months of age (9). The previous recommendation of EBF for four months was changed to 6 months based on accumulative evidence based research by WHO $(10,11)$.

Despite its demonstrated benefits, EBF prevalence and duration in many countries including Ethiopia are lower than the international recommendation of exclusive breastfeeding for the first six months of life $(2,12)$. Based on several studies done in Ethiopia, breastfeeding is nearly universal but the proportion of exclusively breastfed children up to 6 months is less than the optimal recommendations $(13,14)$. The low prevalence of EBF in most developing countries including Ethiopia is attributed to various maternal and child factors such as place of residence, sex and age of the child, mother working outside home, maternal age and educational level, access to mass media and economical status by several researchers $(7,12,15-17)$. The purpose of this article is therefore to document the practice of EBF nationwide and explore whether the previously reported associations elsewhere exist in Ethiopia as well and provide policy makers and NGOs with relevant information for future planning and interventions.

\section{Methods}

The 2005 Ethiopian Demographic and Health Survey (EDHS) was conducted on a nationally representative sample of the nine regions and two city administrations of the country namely: Tigray; Affar; Amhara; Oromiya; Somali; Benishangul-Gumuz; Southern Nations, Nationalities and Peoples (SNNP); Gambela; Harari regions; Addis Ababa and Dire Dawa city administrations. It was conducted from April 27 to August 30, 2005 to provide information on current 
feeding practices as well as other related health and socio-demographic information. A stratified and clustered approach was used to sample the study households. The sample was selected in two stages. In the first stage, 540 clusters were selected from the list of enumeration areas from the 1994 Population and Housing Census sample frame. Of the total representative sample of 14,500 households from 540 clusters selected, only data on women having an infant was considered. The detail of the methodology is available in the EDHS 2005 report (14).

Prior to the analysis, permission was obtained from ORC Macro and the analysis was performed on the basis of children whose age was less than six months, alive and living with the mother at the time of interview that was extracted from the women's data base.

For the assessment of infant breastfeeding, current data were used as defined and recommended by the WHO (8). Current data is information where the mother/care giver is requested to provide information regarding the 24-hour period preceding the survey about the feeding practice of the infant. To estimate the prevalence of exclusive breastfeeding, the proportion of women (with infants aged between 0 and 6 months) who stated to have fed their children only breast milk in the last 24-hours preceding the survey, was expressed as an EBF percentage of the total number of children in the same age group $(8,11)$.

To determine factors influencing the practice of EBF, various maternal and infant characteristics were compared between exclusively breastfed infants and their counterparts. Firstly, a bi-variate analysis was performed to determine the differentials of EBF by explanatory variables. Pearson's chi-square test of independence was performed to test the existence of significant association of risk factors with EBF. Secondly, a stepwise backward logistic regression model was applied to test further the observed significant variables in bi-variate models. A pvalue of less than 0.05 denoted significance in differences. Wealth ranking was grossly categorized into 5 major quintiles based on household assets-lowest (poorest), second (poorer), middle (middle), fourth (richer) and highest (richest).

Since the sampling procedure didn't yield equal probability of selection of the samples, weighting of the samples was applied to produce the national estimate to the descriptive part of this article whereas for the logistic regression models, weighting of the sample was not used. Data were analyzed done SPSS version 13.

The following case definitions were applied, consistent with WHO definitions (8).

- Exclusive breast-feeding: the mother/care giver reported that nothing else but breast milk was given in the last 24 hours preceding the interview
- Predominant breast-feeding: in addition to breast milk, the infant may be receiving water-based drinks including plain water, tea, and soft drink; no food based fluid or milk was allowed within the preceding 24 hours.

- Full breast-feeding: breast-feeding exclusively or predominantly.

- Any breast-feeding: breast-feeding exclusively, predominantly or with any supplements, including milk and solids.

\section{Results}

After weighing of the sample, 1142 women with infants less than six months of age were included in the analysis.

Table 1 shows the characteristics of mothers. As indicated, about three quarters $(70.8 \%)$ of the women were in the age group $20-34$ while $19.9 \%$ were teenagers (15-19 years of age). The majority were Orthodox (41.3\%) followed by Muslim (31.4\%) and Protestant (23.7\%). Although more than 80 ethnic groups participated in the survey, only the five major ethnic groups are presented and distributed as follows; Oromo (34.1\%), Amhara (27.6\%), Tigre (5.5\%), Sidama (4.8\%) and others (28.0\%). The majority of participants were married (95.8\%) and 13 (1.1\%) had never got married. Nearly $93 \%$ of the respondents enrolled were from rural areas while $77(6.7 \%)$ of the participants from urban settings. Nearly a quarter of the women were rich while the poorest and poorer added up to $40 \%$. Only a few $(12.7 \%)$ of the women were reported to work at the time of the interview. A significant proportion (46.0\%) of the respondents were women who had given birth to 2-4 children, followed by multipara mothers (35.3\%) and primipara mothers (18.7\%).

Figure 1 shows the infant feeding practices. As shown, the proportion of women who practiced EBF and predominant breast-feeding (PBF) were $49.0 \%$ and $19 \%$ respectively making the overall rate of full breastfeedings (both EBF and PBF) 68.2\%. The proportion of women who gave pre-lacteal feeding within the first three days of life and used bottle-feeding were $13 \%$ and $28.5 \%$ respectively. Encouragingly, the percentage of women who had never breast-fed was only $0.6 \%$.

Factors associated with EBF are shown in Table 2. As depicted, in the bivariate models, EBF was associated significantly with maternal educational level, current marital status, child age, and economical status. Interestingly no association was observed regarding maternal age, place of residence, current employment of women, and access to mass media, attending antenatal care, and sex of the child.

In the hierarchical models of multivariate analysis, only 3 factors were retained as determinant factors (marital status, wealth index, and child age) for EBF (Table 3). As displayed in the Table, women who are not currently

Ethiop.J.Health Dev. 2009;23(1) 
married were two times more likely to breastfeed their child exclusively than those married $(\mathrm{OR}=2.3$ and $95 \%$ $\mathrm{CI}=1.1,4.7)$. Infants less than two months of age were five times more likely to be on EBF than infant aged four to six months $(\mathrm{OR}=5.0,95 \% \mathrm{CI}=3.5$ to 7.1$)$. Likewise women in the wealth index ranking middle and above were two times more likely to EBF than the reference category (P,0.001).

Table 1: Socio-demographic characteristics of Ethiopian women, 2005

\begin{tabular}{|c|c|c|}
\hline Characteristics & $\mathbf{N}$ & $\%$ \\
\hline \multicolumn{3}{|l|}{ Age } \\
\hline - $15-49$ & 136 & (11.9) \\
\hline - $20-39$ & 808 & (70.8) \\
\hline - $\quad 35-49$ & 197 & $(17.3)$ \\
\hline \multicolumn{3}{|l|}{ Religion } \\
\hline - Orthodox & 471 & $(41.3)$ \\
\hline - $\quad$ Muslim & 358 & $(31.4)$ \\
\hline - Protestant & 271 & $(23.7)$ \\
\hline - Others & 319 & $(28.0)$ \\
\hline \multicolumn{3}{|l|}{ Ethnicity } \\
\hline - Oromo & 390 & (34.1) \\
\hline - Amhara & 315 & $(27.6)$ \\
\hline - $\quad$ Tigre & 63 & $(5.5)$ \\
\hline - Sidama & 55 & $(4.8)$ \\
\hline - Others & 319 & $(28.0)$ \\
\hline \multicolumn{3}{|l|}{ Educational status } \\
\hline - None & 891 & $(78.0)$ \\
\hline - $\quad$ Primary & 205 & $(18.0)$ \\
\hline - Secondary or above & 46 & $(4.0)$ \\
\hline \multicolumn{3}{|l|}{ Current marital status } \\
\hline - Never married & 13 & $(1.1)$ \\
\hline - $\quad$ Married & 1093 & $(95.8)$ \\
\hline - Widowed/divorced/separated & 36 & (3.1) \\
\hline \multicolumn{3}{|l|}{ Place of residence } \\
\hline - Urban & 77 & $(6.7)$ \\
\hline - Rural & 1065 & (93.3) \\
\hline \multicolumn{3}{|l|}{ Wealth index } \\
\hline - Poorest & 228 & (19.9) \\
\hline - $\quad$ Poorer & 228 & $(19.9)$ \\
\hline - $\quad$ Middle & 256 & $(22.4)$ \\
\hline - $\quad$ Richer & 268 & (23.4) \\
\hline - $\quad$ Riches & 163 & $(14.3)$ \\
\hline \multicolumn{3}{|l|}{ Current working status } \\
\hline - Not working & 997 & (87.3) \\
\hline - Working & 145 & $(12.7)$ \\
\hline \multicolumn{3}{|l|}{ Parity } \\
\hline - 1 & 213 & $(18.7)$ \\
\hline - $2-4$ & 525 & $(46.0)$ \\
\hline - 5 and above & 403 & (35.3) \\
\hline
\end{tabular}




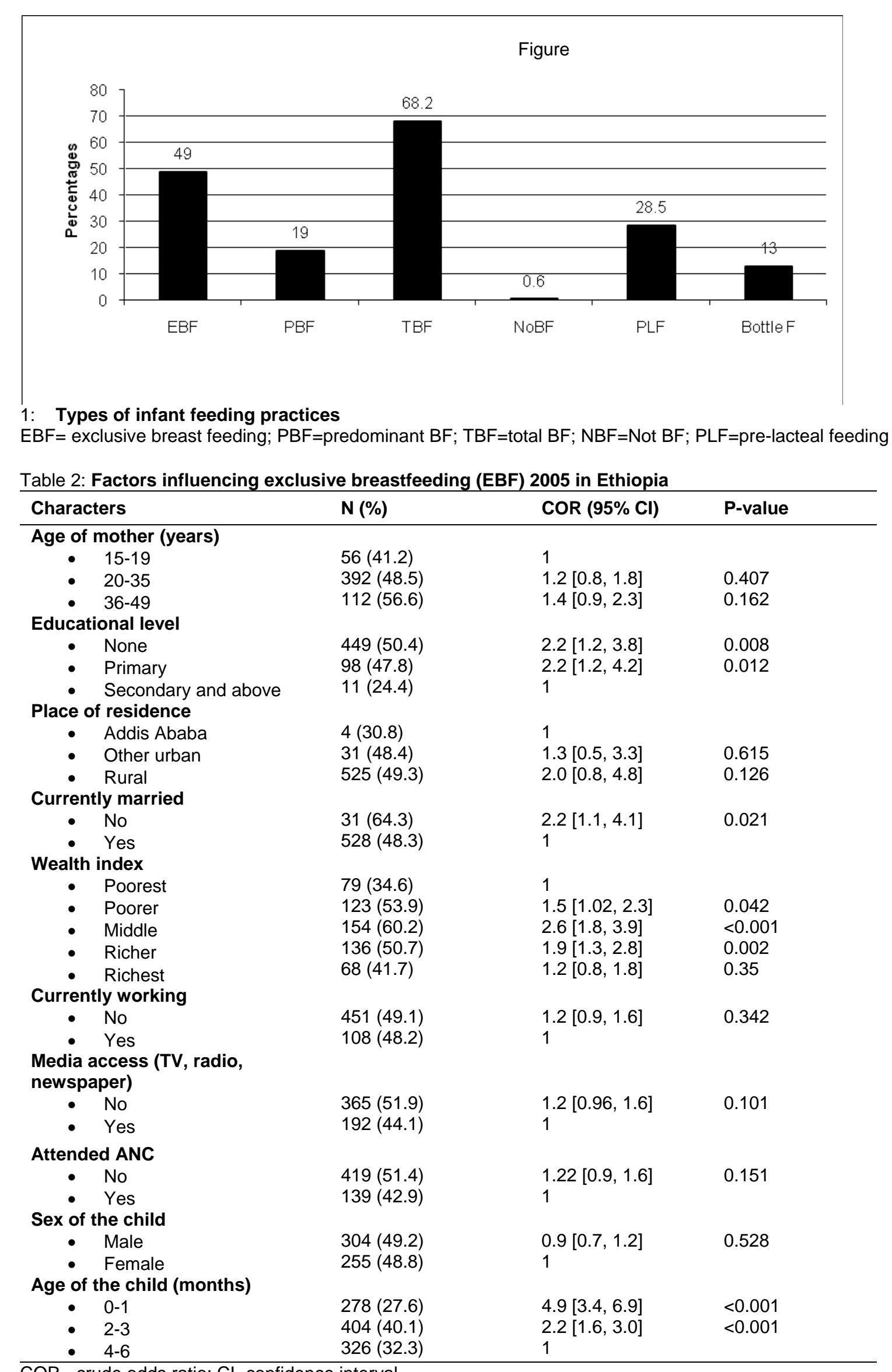

COR $=$ crude odds ratio; $\mathrm{Cl}=$ confidence interval 
Table 3: Logistic regression models showing the determinants of EBF

\begin{tabular}{clll}
\hline Characteristics & N (\% of EBF) & AOR [95\% Cl] & p-value \\
\hline Currently married & $31(64.3)$ & $2.3[1.1,4.7]$ & 0.02 \\
$\bullet \quad$ No & $528(48.3)$ & 1 & \\
Wealth index & & & \\
$\bullet \quad$ Poorest & $79(34.6)$ & 1 & 0.062 \\
$\bullet \quad$ Poorer & $123(53.9)$ & $1.5[0.98,2.3]$ & $<0.001$ \\
$\bullet \quad$ Middle & $154(60.2)$ & $2.9[1.9,4.4]$ & $<0.001$ \\
$\bullet \quad$ Richer & $136(50.7)$ & $2.3[1.5,3.6]$ & 0.006 \\
$\bullet \quad$ Richest & $68(41.7)$ & $2.2[1.3,3.9]$ & \\
Age of the child (months) & & & $<0.001$ \\
$\bullet \quad 0-1$ & $278(27.6)$ & $5.0[3.5,7.1]$ & $<0.001$ \\
$\bullet \quad 2-3$ & $404(40.1)$ & $2.3[1.7,3.3]$ & \\
$\bullet \quad 4-6$ & $326(32.3)$ & 1 & \\
\hline
\end{tabular}

AOR $=$ Adjusted odds ratio

\section{Discussion}

The superiority of breast milk (BM) over any other milk nourishment of the human newborn and infant can hardly be challenged, and over the years it has become more and more apparent that it is the most ideal, safe and complete food that a mother can provide for her newborn. Regrettably, despite the enormous benefits of breast milk, the decline of EBF persists in many developing countries. Efforts made to promote BM use in the past few years, have been encouraging and noteworthy to see mothers swung to EBF in developed countries. Paradoxically enough, this unfavorable trend is noticeable in poor countries where the supply of artificial milk is scarce (2).

In the current study, the prevalence rate of EBF was lower (49.0\%) than the WHO recommendations (2) and was attributed to various maternal and child factors. Nonetheless, when compared with previously reported figures for most developing countries like Tanzania (15), Uganda (19), Kenya (20), Brazil (21), and earlier report in Ethiopia $(12,22)$ the observed figure was similar. Interestingly, full breastfeeding in the country was noted to be a common practice and in conformity with earlier reported research work in Ghana, India and Peru (23). Contrary to our expectation, marital status was found to be associated inversely with EBF (less common among married women) -this inverse relationship appeared to be due to selection factors in the sample and possibly due to the fact that most unmarried women were the teen-ages who were probably under the care of their family which requires further study.

Like in many other developing countries, the practice of mother giving water or tea to their children in addition to the breast milk was common $(20,24)$. Most of the mothers provided their children water because they thought that the milk was insufficient: breast milk seen primarily as food and water is required to satisfy the needs of the child which was probably due to misconception of mothers (24-27). It is evident that early introduction of liquids and solid is unnecessary, reduces the duration and frequency of breastfeeding, and increases risk of infant morbidity and mortality $(16,21)$, and therefore such unhealthy behavior needs to be corrected.

The use of pre-lacteal feeding is another current deterrent impeding the promotion of EBF in many developing countries $(7,19)$ including in Ethiopia as is evidenced by the ongoing practice of feeding other than breast milk within the first three days of newborn. The explanations given in the current study was that mothers believe that they need to wait until the milk started flowing, secondly giving liquid will clean the baby's throat and it has been a long standing tradition (19). When looked at the given explanation, it appears that the habit harms the newborn and exposes him/her to various morbidities and therefore, the behavior needs to be discouraged.

As the age of the child increases the rate of EBF decreased significantly which is again in conformity with some studies done in Uganda, Pakistan and India (7, 16, 29). This could be probably explained by the short birth interval/spacing and other economic factors. It can also be attributed to the fact that post partum care traditionally is given in the first few months when mothers are confined at home, creating an opportunity to exclusively breastfeed their child. The other interesting finding in this study was that higher maternal education level was found to be associated with lower rate of EBF in Ethiopia - this might be explained by the fact that when women are better educated, the opportunity for employment is eminent and thus the opportunity to stay at home and practice EBF is compromised. At the same time, women may be influenced by media advertising milk substitutes. Although the vast majority of women do breast feed their children for a short time, they often cease breast-feeding exclusively too early for the aforementioned reasons. The inverse relationship between maternal level of general education and breastfeeding is also found in other developing countries but is in contra-distinction to the western countries particularly, the USA, where for instance, higher maternal education is related to higher rates of EBF (30), Nonetheless, this association was not 
retained in the logistic regression models because the bivariate analysis is exposed to problems such as confounding. The current situation in Ethiopia may correspond with the period of decline found in Western countries in the middle of the $20^{\text {th }}$ century when the general rise in living standard and wide availability of breast milk substitutes meant that women of higher status chose not to breastfeed. However, strategies need to be developed to avoid repetition of the long period of decline experienced in Western countries, prior to the renaissance in breastfeeding in the 1980s (17).

Factors that interact with the protective effect of breastfeeding include environmental, cultural and economic characteristics. The protective effect of breastfeeding is most important in populations with high infant mortality, high illiteracy, poor sanitation facilities, poor nutritional status, and generally low economic status (31), which fulfill the Ethiopian situation. So in Ethiopia, if optimal breastfeeding is going to be practiced, considerable changes could be achieved in the child morbidity and mortality.

In conclusion, a number of child and maternal attributes were noted to affect the rate of EBF in Ethiopia and therefore, it is recommended that policy makers should look into the issue to give more emphasis in the promotion of EBF through empowerment of women and other corrective measures to the achievement of the fourth Millennium Development Goal.

\section{Reference}

1. Peters E, Wehkamp K-H, Felberbaum RE, ger DK, Linder R. Breastfeeding duration is determined by only a few factors. European Journal of Public Health. 2005;16(2):162-7.

2. Foo LL, Quek SJS, Ng SA, Lim MT, Deurenbergyap M. Breastfeeding prevalence and practices among Singaporean Chinese, Malay and Indian mothers. Health Promotion International. 2005;20(3):229-37.

3. Federal MoH. National Strategy for Child Survival in Ethiopia. Addis Ababa, Ethiopia; 2005.

4. Jones G, Steketee RW, Black RE, Bhutta ZA, Morris SS, Group BCSS. How many child deaths can we prevent this year? The lancet. 2003;362:65-71.

5. Aarts C. Exclusive breastfeeding - Does it make a difference? Uppsala, Sweden: Uppsala University.

6. Yoon PW, Black RE, Moulton LH, Becker S. Effect of Not Breastfeeding on the Risk of Diarrheal and Respiratory Mortality in Children under 2 Years of Age in Metro Cebu, The Philippines. American Journal of Epidemiology. 1996;143:1142-8.

7. Ssenyonga R, Muwonge R, Nankya I. Towards a Better Understanding of Exclusive Breastfeeding in the Era of HIV/AIDS: A Study of Prevalence and Factors Associated with Exclusive Breastfeeding from Birth, in Rakai, Uganda. Journal of Tropical Pediatrics, 2004;50(6):348-53.
8. WHO. Indicators for assessing breast-feeding practices. Report of an informal meeting. Geneva, Seizerland; 11-12 June1991.

9. WHO. Nutrient adequacy of exclusive breastfeeding for the term infant during the first six months of life. Geneva; 2002.

10. WHO. Global strategy for infant and young child feeding. The optimal duration of exclusive breastfeeding. Geneva; 2001.

11. WHO. The optimal duration of exclusive breastfeeding: a systematic review. Geneva; 2002.

12. Haider J, Kloos H,_Haile Mariam D and Demissie T. "Food and Nutrition" In Berhane Y, Haile Mariam D, Kloos H. The Epidemiology and Ecology of Health and Diseases in Ethiopia. 2006. Shama Books: Addis Ababa, Ethiopia.

13. Girma W, Genebo T. Determinants of Nutritional Status of Women and Children in Ethiopia. Calverton, Maryland, USA: ORC Macro; 2002.

14. CSA, ORC M. Ethiopia demographic and health survey 2005. Addis Ababa, Ethiopia and Calverton, Maryland. USA: central statistics agency and ORC Macro; 2006.

15. Simopoulos AP, Grave GD. Factors Associated with the Choice and Duration of Infant-Feeding Practice. Pediatrics. 1984;74:603-14.

16. Shirima R, Greiner T, Kylberg E, Gebre-Medhin M. Exclusive breast-feeding is rarely practised in rural and urban Morogoro, Tanzania. Public Health Nutrition. 2000;4(2):147-54.

17. Morisky DE, Kar SB, Chaudhry AS, Chen KR, Shaheen M, Chickering K. Breast Feeding Practices in Pakistan. Pakistan Journal of Nutrition. 2002;1(3):137-42.

18. Sharief NM, Margolis S, Townsend T. Breastfeeding patterns in Fujairah, United Arab Emirates. Journal of Tropical Pediatrics,. 2001;47:304-6.

19. Engebretsen IMS, Wamani H, Karamagi C, Semiyaga N, Tumwine J, Tylleskär T. Low adherence to exclusive breastfeeding in Eastern Uganda: A community-based cross-sectional study comparing dietary recall since birth with 24-hour recall. BMC Pediatrics. 2007;7(10).

20. Bloss E, Wainaina F, Bailey RC. Prevalence and Predictors of Underweight, Stunting, and Wasting among Children Aged 5 and Under in Western Kenya. Journal of Tropical Pediatrics. 2004;50(5):260-70.

21. Carvalhaes MAdBL, Parada CMGdL, Costa MPd. Factors associated with exclusive breastfeeding in children under four months old in Botucatu-SP, Brazile. Rev Latino-am Enfermagem. 2007;15(1):62-9.

22. Abate G, Kogi-Makau W, Muroki NM. Childfeeding practices as predictors of nutritional status of children in a slum area in Addis Ababa, Ethiopia Ethiop J Health Dev. 1999;13(3):229-38.

23. Bahl R, Frost C, Kirkwood BR, Edmond K, Martines $\mathrm{J}$, Bhandari N, et al. Infant feeding patterns and risk

Ethiop.J.Health Dev. 2009;23(1) 
of death and hospitalization in the first half of infancy: multicentre cohort study. Bulletin of World Health Organization. 2005;83(6):418-26.

24. Brunken GS, Silva SM, França GVA, Escuder MM, Venâncio SI. Risk factors for early interruption of exclusive breastfeeding and late introduction of complementary foods among infants in midwestern Brazil. Jornal de Pediatria. 2006;82(6):445-51.

25. Shiva F, Nasiri M. A Study of Feeding Patterns in Young Infants. Journal of Tropical Pediatrics. 2003;49(2):89-92.

26. Parada CMGdL, Carvalhaes MAdBL, Jamas MT. Complementary feeding practices to children during their first year of life. Rev Latino-am Enfermagem. 2007;15(2):282-9.

27. Nwankwo BO, Brieger WR. Exclusive breastfeeding is undermined by use of other liquids in rural Southwestern Nigeria. Journal of Tropical Pediatrics. 2002;48:109-12.
28. Paoli Md, Manongi R, Helsing E, Klepp K-I. Exclusive Breastfeeding in the Era of AIDS. J Hum Lact. 2001;17(4):313-20.

29. Sachdev HPS, Mehrptra S. Predictors of exclusive breastfeeding in early infancy: operational implications. Indian Pediatrics. 1995;32:1277-96.

30. Ryan AS. The resurgence of breastfeeding in United States. Pediatrics. 1997:99:E12.

Mihrshahi S, Ichikawa N, Shuaib M, Oddy W, Ampon R, Dibley MJ, et al. Prevalence of Exclusive Breastfeeding in Bangladesh and Its Association with Diarrhoea and Acute Respiratory Infection: Results of the Multiple Indicator Cluster Survey 2003. J Health Popul Nutr 2007 Jun;25(2):195-204. 